\title{
Radiation and Admittance of an Insulated Slotted-Sphere Antenna Surrounded by a Strongly Ionized Plasma Sheath ${ }^{1}$
}

\author{
John W. Marini \\ (February 29, 1960; revised April 1, 1960)
}

\begin{abstract}
Given the voltage distribution along the slot, expressions for the radiation pattern, input admittance, and the external efficiency of an insulated slotted-sphere antenna surrounded by a homogeneous, isotropic, strongly ionized sheath are obtained.

At low frequencies the input impedance is proportional to the sum of the intrinsic impedance of the sheath and an equivalent inductance due to the insulating coating, the radiation pattern reduces to that of a small loop, while the external efficiency is the product of three factors arising because of the power dissipated in the sheath by higher order modes that contribute little to the radiation field, attenuation through the sheath of the modes that do radiate, and reflection loss of these modes at the outer surface of the sheath.

Since the reflection loss decreases with increasing frequency while the attenuation increases, there exists an optimum frequency of operation. At this frequency, the ionized sheath has a thickness equal to two-and-one-half skin depths.
\end{abstract}

\section{Introduction}

In connection with the re-entry of a space vehicle into the earth's atmosphere, a knowledge of the characteristics of a transmitting antenna in its prevailing environment is important in order to achieve the most effective design for efficient radiation. Particularly important is the behavior of an antenna in a strongly ionized medium.

To study the behavior of such an antenna analytically, it is natural to represent the vehicle, antenna, and surrounding mediums by means of simple geometric surfaces leading to mathematical expressions that can be handled readily. One such surface is the cylinder, which has been used by Wait to study a number of radiating structures including the dielectric-clad slotted-cylinder antenna radiating into free space $[1] .^{2}$

The spherical surface also yields simple, tractable models for initial studies of the subject. The slottedsphere antenna radiating into free space has been treated by Mushiake and Webster [2].

The model used in this paper is an insulated slottedsphere antenna surrounded by an homogeneous, uniform, strongly ionized plasma sheath. Expressions for the input admittance and radiation characteristics of the antenna are derived. While the model adopted does not duplicate the shape of the space vehicle and the surrounding sheath, the analysis nevertheless sheds light on the physical processes involved and provides approximate formulas for estimating some quantities of interest. The assumption that the sheath is homogeneous is not a good one for the physical situation under consideration, but

${ }_{1}^{1}$ Contribution from Electromagnetic Research Corporation, Washington 5 D.C. The research reported in this paper was supported by the Missile and Space Vehicles Department of the General Electric Company under a Departmace vehicles Department of the

2 Figures in brackets indicate the literature references at the end of this paper. was made for the sake of mathematical simplicity and in the absence of information about the gradients existing in the sheath.

The conditions existing in the sheath surrounding a space vehicle during re-entry are not well known at present. If one assumes, however, that a temperature of $5,000^{\circ} \mathrm{K}$, a particle density of $10^{23}$ per cubic meter and an electron density $\mathrm{N}$ of $10^{19}$ per cubic meter are representative [3], then simple calculations [4] give a collision frequency $\nu \sim 10^{9}$ per second and a plasma frequency $\omega_{N} \sim 10^{11}$ per second. The gyromagnetic frequency due to the earth's magnetic field is roughly $2 \pi \times 10^{6}$ radians per second, which is much less than the value calculated for the collision frequency. Inspection of the usual formula for the index of refraction given in magneto-ionic theory [5] shows that the effect of the earth's magnetic field on the values obtained for the index of refraction is very small in such cases. Consequently it will be assumed that the sheath can be regarded as an isotropic medium with an index of refraction given by $n^{2} \simeq 1-\left(\omega_{N} / \omega\right)^{2} /(1-i \nu / \omega)$ where $\omega$ is the radian frequency of the signal emitted by the antenna.

If the transmitted frequency $\omega / 2 \pi$ can be made sufficiently large, $n$ reduces to unity and the operation of the antenna is not affected by the presence of the sheath. On the other hand, over a wide range of frequencies, both $\nu / \omega$ and $\omega_{N}^{2} / \omega \nu$ are much greater than unity and the index of refraction is given by $n \simeq \omega_{N}^{2} / i \omega \nu$. In this case the medium behaves like a good conductor with a conductivity $\sigma=\epsilon_{0} \omega_{N}{ }^{2} / \nu=$ $9 \pi \times 10^{-9} N / \nu$. The treatment here is confined to this range of frequencies.

In the frequency range considered, then, it is to be expected that much of the power supplied to the antenna will be dissipated through ohmic losses in the conducting sheath. The electromagnetic field established by the spherical antenna can be decom- 
posed into the sum of spherical waves corresponding to various spherical modes. Because of the conductivity of the ionized sheath, these spherical waves are attenuated as they progress through the sheath. When the waves reach the outer boundary of the sheath, reflection takes place, and the amplitudes of the waves transmitted into free space are further reduced.

It is important to compare the magnitudes of the effects of attenuation and reflection on the transmitted waves. The attenuation of all lower order modes is essentially equal to that of a plane wave propagating through a medium having the same electromagnetic properties as the sheath and the same thickness. Being exponential, it is the more significant loss over most of the frequency range under consideration. Measured in decibels, the attenuation is directly proportional to the square root of the transmitter frequency. It follows that attenuation loss is reduced by lowering the frequency, at least in the range in which the sheath acts like a good conductor.

In contrast, reduction in amplitude of the transmitted waves due to poor transmission through the outer boundary of the sheath increases as the frequency is lowered. Eventually, then, an optimum frequency is reached which represents the best compromise between attenuation and reflection loss.

In view of the necessity to operate at a fairly low frequency to minimize attenuation loss, it is logical to consider the case where the frequency is so low that the sphere is a small antenna, i.e., that it has a radius that is small compared to the free-space wavelength. In this case, simple expressions are obtained for the input impedance of the slot, the radiation pattern, the external efficiency of the antenna, and the optimum frequency of operation.

In fact, for a small antenna, the reflection of a given mode at the outer boundary of the sheath increases strongly with the order of the mode, so that the radiation pattern is due only to the lowest order mode and is essentially the pattern of a small current loop. Since the higher order modes contribute little to the radiation field in this case, the expression obtained for the ratio of power radiated by the slot to that delivered to the slot turns out to be the product of three factors, one representing the ratio of power supplied to the lowest order mode to that supplied to all modes, the second representing attenuation of the lowest order mode in its passage through the sheath, and the third representing the transmission coefficient for the latter mode at the outer boundary of the sheath.

In all cases it is necessary that the antenna be provided with an insulating cover in the immediate vicinity of the feed in order that the assumed voltage or current distribution is realized and that conduction losses in the ionized medium not be exorbitant. The case treated here is that of an insulating layer which covers the entire spherical surface. A criterion is established for the thickness of this insulating layer.

\section{Formulation of the Problem}

Consider a perfectly conducting sphere of radius $a$ covered with a dielectric coating of outer radius $b$, which in turn is surrounded by an ionized sheath of outer radius $c$. On the sphere, a narrow slot of length $2 l$ (measured along the surface of the sphere) and of width $2 s$ is located with its center at $\theta=\pi / 2$, $\phi=0$ and its ends at $\theta=\pi / 2, \phi= \pm l / a$ as shown in figure 1.

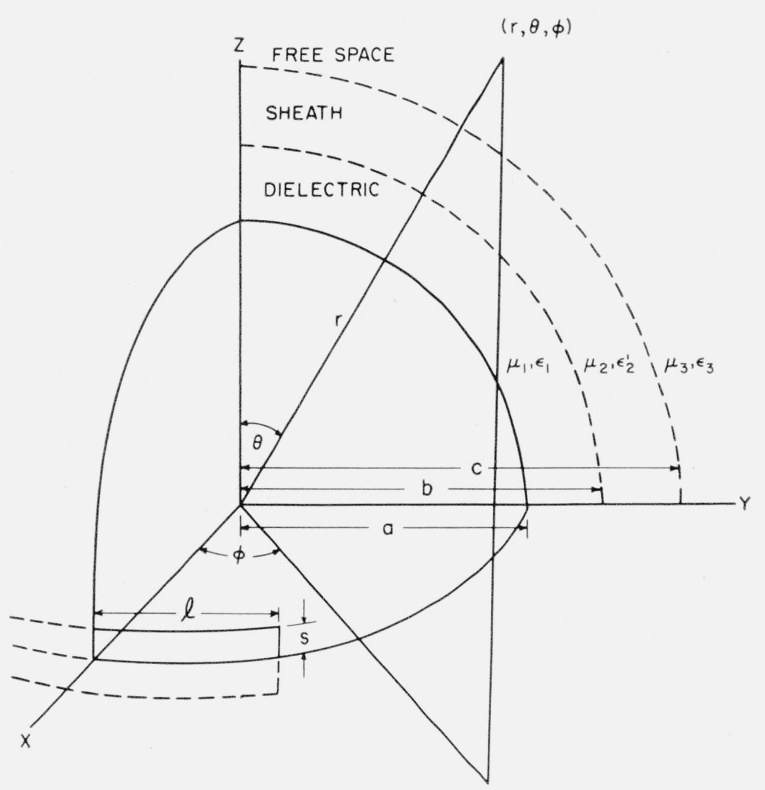

FiguRE 1. Insulated slotted-sphere antenna.

The problem under consideration is to determine the exterior admittance of the slot, assuming that it is fed at the center, and to obtain the radiation field.

The outer medium is assumed to be free space. The electromagnetic properties of the dielectric coating will be characterized by its permeability $\mu_{1}$, and its complex dielectric constant $\epsilon_{1}^{\prime}$. Since the coating is assumed to have no conductivity, the complex dielectric constant is real and equal to the ordinary dielectric constant, $\epsilon_{1}^{\prime}=\epsilon_{1}$. The properties of the ionized sheath are characterized by the constants $\mu_{2}$ and $\epsilon_{2}^{\prime}$. In this case, however, the medium has finite conductivity, and the complex dielectric constant $\epsilon_{2}^{\prime}$ is equal to $\epsilon_{2}+(\sigma / i \omega)$ where $\epsilon_{2}$ is the equivalent dielectric constant and $\sigma$ the equivalent conductivity of the ionized layer. The permeability, $\mu_{2}$, of the ionized region is the same as that of free space, $\mu_{0}$. It has been assumed that the ionized sheath acts like a good conductor. Thus $\sigma$ is large compared to $\omega \epsilon_{2}$, so that $\epsilon_{2}^{\prime} \approx \sigma / i \omega$.

Use will be made of the constants $k=\omega \sqrt{\mu \epsilon^{\prime}}$ and $\eta=\sqrt{\mu / \epsilon^{\prime}}$, ik being the complex propagation constant and $\eta$ the complex impedance of the medium to plane waves. In the dielectric and in free space 
both of these constants will be real, while in the ionized sheath $k_{2} \approx \sqrt{\omega \mu_{2} \sigma} e^{-i \pi / 4}$ and $\eta_{2} \approx \sqrt{\omega \mu_{2} / \sigma} e^{i \pi 4 /}$. Rationalized MKS units and a time variation $e^{+i \omega t}$ are employed.

A formal solution of the exterior electromagnetic boundary value problem for a sphere has been given by Bailin and Silver [6]. Written below is a formal solution obtained using their procedure under the assumption that the impressed electric intensity $E_{\theta}(a, \theta, \phi)$ on the surface of the sphere is an even function of $\phi$ (since the slot is fed at the center) and that the impressed electric intensity $E_{\phi}(a, \theta, \phi)$ is negligible. The functions employed differ from those used by Bailin and Silver, however, and the constants obtained differ by a common factor.

Using the index $\lambda=1,2,3$ to refer to the dielectric, the sheath, and free space, respectively, the formal solution for the electromagnetic field is

$$
\left.\begin{array}{l}
E_{r}^{\lambda}=\left(\frac{\partial^{2}}{\partial r^{2}}+k_{\lambda}^{2}\right) \Pi^{E \lambda} \\
E_{\theta}^{\lambda}=\frac{1}{r} \frac{\partial^{2}}{\partial r \partial \theta} \Pi^{E \lambda}-i \omega \mu_{\lambda} \frac{1}{r \sin \theta} \frac{\partial}{\partial \phi} \Pi^{H \lambda} \\
E_{\phi}^{\lambda}=\frac{1}{r \sin \theta} \frac{\partial^{2}}{\partial r \partial \theta} \Pi^{E \lambda}+i \omega \mu_{\lambda} \frac{1}{r} \frac{\partial}{\partial \theta} \Pi^{H \lambda} \\
H_{r}^{\lambda}=\left(\frac{\partial^{2}}{\partial r^{2}}+k_{\lambda}^{2}\right) \Pi^{H \lambda} \\
H_{\theta}^{\lambda}=i \omega \epsilon_{\lambda}^{\prime} \frac{1}{r \sin \theta} \frac{\partial}{\partial \phi} \Pi^{E \lambda}+\frac{1}{r} \frac{\partial^{2}}{\partial r \partial \theta} \Pi^{H \lambda} \\
H_{\phi}^{\lambda}=-i \omega \epsilon_{\lambda}^{\prime} \frac{1}{r} \frac{\partial}{\partial \theta} \Pi^{E \lambda}+\frac{1}{r \sin \theta} \frac{\partial^{2}}{\partial r \partial \phi} \Pi^{H \lambda}
\end{array}\right\}
$$

with

$$
\left.\begin{array}{r}
\Pi^{E \lambda}=\sum_{n=1}^{\infty} \sum_{m=0}^{n} A_{n m}^{E} Z_{n}^{E \lambda}\left(k_{\lambda} r\right) \bar{P}_{n}^{m}(\cos \theta) \cos m \phi \\
\Pi^{H \lambda}=\sum_{n=1}^{\infty} \sum_{m=0}^{n} A_{n m}^{H} Z_{n}^{H \lambda}\left(k_{\lambda} r\right) \bar{P}_{n}^{m}(\cos \theta) \sin m \phi
\end{array}\right\}
$$

and

$$
\left.\begin{array}{rl}
A_{n m}^{E} & =-\frac{1}{\left(1+\delta_{o m}\right) n(n+1)} \frac{a}{k_{1} \pi} \frac{1}{Z_{n}^{E 1^{\prime}}\left(k_{1} a\right)} \\
\int_{-\pi}^{\pi} \int_{0}^{\pi} E_{\theta}(a, \theta, \phi) \bar{P}_{n}^{m^{\prime}}(\cos \theta) \sin ^{2} \theta \cos m \phi d \theta d \phi & \\
A_{n m}^{H}=-\frac{1}{\left(1+\delta_{o m}\right) n(n+1)} \frac{a}{\pi} \frac{1}{i \omega \mu_{1} Z_{n}^{H 1}\left(k_{1} a\right)} \\
\cdot \int_{-\pi}^{\pi} \int_{0}^{\pi} m E_{\theta}(a, \theta, \phi) \bar{P}_{n}^{m}(\cos \theta) \cos m \phi d \theta d \phi .
\end{array}\right\}
$$

The superscripts $E$ and $H$ refer to electric and magnetic type modes, respectively. The constant $\delta_{0 m}=1$ if $m=0 ; \delta_{0 m}=0$ if $m \neq 0$. The functions $\bar{P}_{n}^{m}$ $(\cos \theta)$ are normalized associated Legendre polynomials [7] while the radial functions $Z_{n}^{E \lambda}(\kappa \lambda r)$ and $Z_{n}^{H \lambda}(\kappa \lambda r)$ are linear combinations of the functions

$$
\begin{aligned}
& j_{n}(z)=\sqrt{\pi z / 2} J_{n+1 / 2}(z) \\
& h_{n}^{(1)}(z)=\sqrt{\pi z / 2} H_{n+1 / 2}^{(1)}(z) \\
& h_{n}^{(2)}(z)=\sqrt{\pi z / 2} H_{n+1 / 2}^{(2)}(z)
\end{aligned}
$$

where $\mathbf{J}_{n+1 / 2}(z), \quad H_{n+1 / 2}^{(1)}(z)$ and $H_{n+1 / 2}^{(2)}(z)$ are the usual Bessel and Hankel functions or order $n+1 / 2$ [8].

The voltage distribution across the slot is assumed to be known. Since the slot is fed at the center, the distribution is an even function of the azimuthal angle $\phi$, and can be expanded in a Fourier cosine series

$$
V(\phi)=\sum_{p=0}^{\infty} V_{p} \cos p \phi .
$$

The electric intensity tangent to the surface of the sphere is, then,

$$
\begin{aligned}
& E_{\theta}^{1}(a, \theta, \phi)=\left\{\begin{array}{l}
\sum_{p=0}^{\infty} \frac{V_{p} \cos p \phi}{2 s}, \frac{\pi}{2}-\frac{s}{a}<\theta<\frac{\pi}{2}+\frac{s}{a} \\
0 ; \quad \text { otherwise }
\end{array}\right. \\
& E_{\phi}^{1}(a, \theta, \phi)=0 .
\end{aligned}
$$

\section{Formal Solution}

The equations given in section 2 above can be used to derive formal expressions for the input admittance and radiation field of the slotted-sphere antenna of figure 1. In this section, the procedure for obtaining these expressions is given.

\subsection{Input Admittance}

To obtain a formal expression for the input admittance is a fairly straightforward procedure, Equation (6) is substituted into (3) to obtain $A_{n m}^{E}$ and $A_{n m}^{H}$ :

$$
A_{n m}^{E}=-\frac{V_{m}}{k_{1} Z_{n}^{E 1^{\prime}}\left(k_{1} a\right)} \frac{\bar{P}_{n}^{m \prime}(0)}{n(n+1)} \cdot I_{E}
$$

where

$$
I_{E}=\frac{a}{2 s} \int_{\pi / 2-s / a}^{\pi / 2+s / a} \frac{\bar{P}_{n}^{m^{\prime}}(\cos \theta)}{\bar{P}_{n}^{m}(0)} \sin ^{2} \theta d \theta
$$

and

where

$$
A_{n m}^{H}=-\frac{V_{m}}{i \omega \mu_{1} Z_{n}^{H 1}\left(k_{1} a\right)} \frac{m \bar{P}_{n}^{m}(0)}{n(n+1)} \cdot I_{H}
$$

$$
I_{H}=\frac{a}{2 s} \int_{\frac{\pi}{2}-\frac{s}{a}}^{\pi / 2+s / a} \frac{\bar{P}_{n}^{m}(\cos \theta)}{\bar{P}_{n}^{m}(0)} d \theta
$$


$I_{E}$ and $I_{H}$ are approximately equal to unity for narrow slots and values of $n$ that are not too large.

Equations (7a) and (7b) now are substituted into (2) to obtain $\Pi^{E I}$ and $\Pi^{H I}$, and these in turn are used to obtain $H_{\phi}^{1}$ from (1). The input admittance is obtained by equating the conjugate of the complex power supplied to the slot to that radiated by the slot.

$$
Y|V(0)|^{2}=\int_{-\pi}^{\pi} \int_{0}^{\pi} E_{\theta}^{*}(a, \theta, \phi) H_{\phi}(a, \theta, \phi) a^{2} \sin \theta d \theta d \phi .
$$

Here the integration can be taken over the entire sphere since this must give the same result as integration over the face of the slot alone $\left(E_{\theta}\right.$ being zero elsewhere). $E_{\theta}^{*}$ is the complex conjugate of $E_{\theta}$, and is obtained from eq (6). This procedure gives a double series expansion for the input admittance.

$$
\begin{aligned}
Y=\sum_{n=1}^{\infty} \sum_{m=0}^{n} i\left|\frac{V_{m}}{V(0)}\right|^{2} \frac{\left(1+\delta_{v m}\right) \pi}{\eta_{1}}\left\{\frac{Z_{n}^{H 1}\left(k_{1} a\right)}{Z_{n}^{H 1}\left(k_{1} a\right)}\right. \\
\\
\left.\frac{m^{2} \overline{P_{n}^{m}}(0)^{2} I_{H}^{2}}{n(n+1)}-\frac{Z_{n}^{E 1}\left(k_{1} a\right)}{Z_{n}^{E 1^{\prime}}\left(k_{1} a\right)} \frac{\overline{P_{n}^{m}}(0)^{2} I_{E}^{2}}{n(n+1)}\right\} .
\end{aligned}
$$

Equation (8) is exact if the impressed voltage distribution given by (6) is realized and if the radial functions are known. ${ }^{3}$

\subsection{Determination of the Radial Functions}

Since the voltage distribution across the slot is assumed known, the only quantities left to determine in eq (8) are the radial functions, $Z_{n}^{E 1}\left(k_{1} r\right)$ and $Z_{n}^{H 1}\left(k_{1} r\right)$. They are determined from the boundary conditions of continuity of the tangential components of the electric and magnetic intensities at the inner and outer boundaries of the sheath, and by the radiation condition at infinity. If the sheath is sufficiently thick, it may be assumed that waves reflected from the boundary $c$ between the sheath and free space can be neglected at the boundary $b$ between the sheath and the dielectric. This assumption is not necessary, but it is a good one for the physical situation considered, and it simplifies the expressions obtained. Under this assumption, the input admittance is the same as if the ionized sheath were of infinite thickness.

\section{a. Determination of $Z_{n}^{E 1}$ and $Z_{n}^{H 1}$}

Treating the electric type modes first, in the dielectric let

$$
Z_{n}^{E 1}\left(k_{1} r\right)=H_{n}^{(2)}\left(k_{1} r\right)-R_{n}^{E 1} j_{n}\left(k_{1} r\right)
$$

which represents the superposition of an outgoing and a standing wave ${ }^{4}$ resulting from reflection, and

\footnotetext{
${ }^{3}$ In the case treated by Muskiake and Webster of an uninsulated slotted sphere antenna radiating into free space these functions are simply the functions $h_{n}^{(2)}$; defined in eq (4).

${ }^{4}$ The function $h_{n}^{(1)}\left(k_{1} r\right)$ which represents a reflected wave can, of course, be used instead of $j_{n}\left(k_{1} r\right)$, but the latter turns out to be more convenient when the functions are approximated for small values of the argument since it turns out that only one term in the series expansion for $j_{n}\left(k_{1} r\right)$ needs to be retained, while two are needed if $h_{n}^{(1)}\left(k_{1} r\right)$ is used.
}

in the sheath let

$$
Z_{n}^{E 2}\left(k_{2} r\right)=T_{n}^{E 1} h_{n}^{(2)}\left(k_{2} r\right)
$$

which represents an outgoing wave only, in accordance with the assumption above that waves reflected from the boundary at $c$ may be neglected at $b$. $R_{n}^{E 1}$ and $T_{n}^{E 1}$ are constants which must be chosen to satisfy the boundary conditions at the intertace between the insulating layer and the ionized sheath. The boundary conditions will be met if $k_{\lambda} Z_{n}^{E \lambda}\left(k_{\lambda} r\right)$ and $\epsilon_{\lambda}^{\prime} Z_{n}^{E \lambda}\left(k_{\lambda} r\right)$ are continuous at the boundary. Therefore we have

$$
\begin{aligned}
& k_{1} h_{n}^{(2) \prime}\left(k_{1} b\right)-R_{n}^{E 1} k_{1} j_{n}^{\prime}\left(k_{1} b\right)=T_{n}^{E 1} k_{2} h_{n}^{(2) \prime}\left(k_{2} b\right) \\
& \epsilon_{1} h_{n}^{(2)}\left(k_{1} b\right)-R_{n}^{E 1} \epsilon_{1} j_{n}\left(k_{1} b\right)=T_{n}^{E 1} \epsilon_{2}^{\prime} h_{n}^{(2)}\left(k_{2} b\right) .
\end{aligned}
$$

Solving for $R_{n}^{E 1}$ and $T_{n}^{E 1}$

$$
\begin{aligned}
& R_{n}^{E 1}=\frac{k_{1} \epsilon_{2}^{\prime} h_{n}^{(2) \prime}\left(k_{1} b\right) h_{n}^{(2)}\left(k_{2} b\right)-k_{2} \epsilon_{1} h_{n}^{(2)}\left(k_{1} b\right) h_{n}^{(2) \prime}\left(k_{2} b\right)}{k_{1} \epsilon_{2}^{\prime} j_{n}^{\prime}\left(k_{1} b\right) h_{n}^{(2)}\left(k_{2} b\right)-k_{2} \epsilon_{1} j_{n}\left(k_{1} b\right) h_{n}^{(2) \prime}\left(k_{2} b\right)} \\
& T_{n}^{E 1}=\frac{k_{1} \epsilon_{1}\left[j_{n}^{\prime}\left(k_{1} b\right) h_{n}^{(2)}\left(k_{1} b\right)-j_{n}\left(k_{1} b\right) h_{n}^{(2) \prime}\left(k_{1} b\right)\right]}{k_{1} \epsilon_{2}^{\prime} j_{n}^{\prime}\left(k_{1} b\right) h_{n}^{(2)}\left(k_{2} b\right)-\left(k_{2} \epsilon_{1} j_{n}\left(k_{1} b\right) h_{n}^{(2)}\left(k_{2} b\right)\right.} .
\end{aligned}
$$

The expression above for $T_{n}^{E 1}$ may be simplified by using the Wronskian,

$$
j_{n}^{\prime}\left(k_{1} b\right) h_{n}^{(2)}\left(k_{1} b\right)-j_{n}\left(k_{1} b\right) h_{n}^{(2) \prime}\left(k_{1} b\right)=i .
$$

The radial functions $Z_{n}^{H 1}\left(k_{1} r\right)$ and $Z_{n}^{H 2}\left(k_{2} r\right)$ for the magnetic type modes may be determined in the same manner. Thus let

$$
\begin{aligned}
& Z_{n}^{H 1}\left(k_{1} r\right)=h_{n}^{(2)}\left(k_{1} r\right)-R_{n}^{H 1} j_{n}\left(k_{1} r\right) \\
& Z_{n}^{H 2}\left(k_{2} r\right)=T_{n}^{H 1} h_{n}^{(2)}\left(k_{2} r\right) .
\end{aligned}
$$

In this case the boundary conditions are satisfied if $k_{\lambda} Z_{n}^{H \lambda^{\prime}}\left(k_{\lambda} r\right)$ and $\mu_{\lambda} Z_{n}^{H \lambda}\left(k_{\lambda} r\right)$ are continuous at the boundary. Comparing the boundary conditions for the electric and magnetic cases, it is evident that the expressions for $R_{n}^{H 1}$ and $T_{n}^{H 1}$ are the same as those for $R_{n}^{E 1}$ and $T_{n}^{E 1}$ given in eq (10) but with $\mu_{\lambda}$ replacing $\epsilon_{\lambda}^{\prime}$ (or $\epsilon_{\lambda}$ where $\epsilon_{\lambda}=\epsilon_{\lambda}^{\prime}$ ) throughout.

With the radial functions $Z_{n}^{E 1}$ and $Z_{n}^{H 1}$ given by eq (9), (10), and (11), eq (8) provides a formal solution to the problem of an insulated slotted sphere antenna radiating into an electrically thick conducting layer.

\section{b. Determination of $Z_{n}^{E 3}\left(k_{3} r\right)$ and $Z_{n}^{H 3}\left(k_{3} r\right)$}

In order to obtain the radiation field from (1), (2), and (3), the functions $Z_{n}^{E 3}\left(k_{3} r\right)$ and $Z_{n}^{H 3}\left(k_{3} r\right)$ must be determined. Let

$$
\begin{aligned}
& Z_{n}^{E 2}\left(k_{2} r\right)=T_{n}^{E 1}\left[h_{n}^{(2)}\left(k_{2} r\right)-R^{E 2} h_{n}\left(k_{2} r\right)\right] \\
& \mathrm{Z}_{n}^{E 3}\left(k_{3} r\right)=T_{n}^{E 1}\left[T_{n}^{E 2} h_{n}^{(2)}\left(k_{3} r\right)\right] .
\end{aligned}
$$


The first term in the upper equation represents the outgoing wave given in $(9 \mathrm{~b})$. The second term represents the reflected wave which was neglected in $(9 \mathrm{~b})$. The lower equation represents an outgoing wave only, in order to satisfy the radiation condition at infinity. The procedure for determining $T_{n}^{E 2}$ is the same as that used to determine $T_{n}^{E 1}$, except that the function $h_{n}^{(1)}$ replaces the function $j_{n}$. Consequently

$$
T_{n}^{E 2}=\frac{k_{2} \epsilon_{2}^{\prime}\left[h_{n}^{(1) \prime}\left(k_{2} c\right) h_{n}^{(2)}\left(k_{2} c\right)-h_{n}^{(1)}\left(k_{2} c\right) h_{n}^{(2) \prime}\left(k_{2} c\right)\right]}{k_{2} \epsilon_{3} h_{n}^{(1) \prime}\left(k_{2} c\right) h_{n}^{(2)}\left(k_{3} c\right)-k_{3} \epsilon_{2}^{\prime} h_{n}^{(1)}\left(k_{2} c\right) h_{n}^{(2) \prime}\left(k_{3} c\right)}
$$

The Wronskian in the numerator in this case is equal to $2 i$. $T_{n}^{H 2}$ can be obtained from (13) by substituting $\mu_{\lambda}$ for $\epsilon_{\lambda}^{\prime}$.

\subsection{Radiation Field}

With the radial functions in free space determined from (12) and (13) and from the corresponding expressions for the magnetic case, the radiation field can be derived from (1), (2), (3), and (4). The $\theta$ and $\phi$ components of the electric intensity in free space are

$$
\begin{aligned}
E_{\theta}^{(3)}=\sum_{n=1}^{\infty} \sum_{m=0}^{n}\{- & k_{3} A_{n m}^{E} T_{m}^{E 1} T_{n}^{E 2} \frac{h_{n}^{(2)}\left(k_{3} r\right)}{r} \\
\times \bar{P}_{n}^{m \prime}(\cos \theta) & \sin \theta \cos m \phi-i \omega \mu_{3} A_{n m}^{H} T_{n}^{H 1} T_{n}^{H 2} \\
& \left.\times \frac{h_{n}^{(2)}\left(k_{3} r\right)}{r} \frac{\bar{P}_{n}^{m}(\cos \theta)}{\sin \theta} m \cos m \phi\right\}
\end{aligned}
$$$$
E_{\phi}^{(3)}=\sum_{n=1}^{\infty} \sum_{m=0}^{n}\left\{-k_{3} A_{n m}^{E} T_{n}^{E 1} T_{n}^{E 2} \frac{h_{n}^{(2)}{ }^{\prime}\left(k_{3} r\right)}{r}\right.
$$

$$
\begin{array}{r}
\times \frac{\bar{P}_{n}^{m}(\cos \theta)}{\sin \theta} m \sin m \phi-i \omega \mu_{3} A_{n m}^{H} T_{n}^{H 1} T_{n}^{H 2} \\
\left.\times \frac{h_{n}^{(2)}\left(k_{3} r\right)}{r} \bar{P}_{n}^{m^{\prime}}(\cos \theta) \sin \theta \sin m \phi\right\} .
\end{array}
$$

The radiation field is obtained from the expressions above by replacing $h_{n}^{(2)}\left(k_{3} r\right)$ and $h_{n}^{(2) \prime}\left(k_{3} r\right)$ by their asymptotic expansions for large $r$ :

$$
h_{n}^{(2)}\left(k_{3} r\right) \sim e^{-i\left(k_{3} r-\frac{n+1}{2} \pi\right)} ; h_{n}^{(2) \prime}\left(k_{3} r\right) \sim-i e^{-i\left(k_{3} r-\frac{n+1}{2} \pi\right) .}
$$

Also, for large $r, H_{\theta}^{(3)} \approx-E_{\phi}^{(3)} / \eta_{3}$ and $H_{\phi}^{(3)} \approx E_{\theta}^{(3)} / \eta_{3}$.

The radiation field so determined is exact for an insulated slotted sphere antenna radiating through an electrically thick sheath.

\section{Approximate Solution}

The expressions for the input admittance and the radiation field can be greatly simplified in the case where the sphere is a small antenna. If the frequency is sufficiently low, then the distance $b$ is small compared to the wavelength in the dielectric, and $c$ is small compared to the wavelength in free space. The conductivity of the sheath has already been assumed large so that $b$ is a large number of skin depths. Then $k_{1} a, k_{1} b$, and $k_{3} c$ are small while $k_{2} b$ and $k_{2} c$ are large. Functions of $k_{1} a, k_{1} b$ and $k_{3} c$ consequently can be replaced by their approximations for small arguments, while functions of $k_{2} b$ and $k_{2} c$ can be replaced by their asymptotic expansions for large arguments. It will be assumed that the insulating layer is thin, i.e., that $(b-a) / b \ll 1$.

\subsection{Input Admittance}

When these approximations are made the input admittance eventually reduces [9] to

$$
\begin{gathered}
Y \approx \sum_{n=0}^{\infty} \sum_{m=0}^{n}\left(1+\delta_{o m}\right) \pi\left\{\frac{1}{n_{2}+i \omega \mu_{1}(b-a)} \frac{m^{2}}{n(n+1)} \bar{P}_{n}^{m}(0)^{2}\right. \\
\left.+\frac{1}{\eta_{2}+\frac{n(n+1)(b-a)}{i \omega \epsilon_{1} a b}} \frac{\bar{P}_{n}^{m^{\prime}(0)}}{n(n+1)}\right\}\left|\frac{V_{m}}{V(0)}\right|^{2} \cdot \quad \text { (15) }
\end{gathered}
$$

In form, eq (15) represents the total admittance of two types of circuits in parallel, one circuit corresponding to each mode. The first type of circuit contains the surface impedance $\eta_{2}$ of the ionized sheath in series with an equivalent inductance $\mu_{1}(b-a)$. This circuit apparently is associated with the currents flowing on the surface of the sphere together with those induced on the interface between the dielectric and ionized layers. The second type of circuit also contains the surface impedance $\eta_{2}$, this time, however, in series with an equivalent capacitance $C=\epsilon_{1} a b / n(n+1)(b-a)$ between the sphere and the interface. This circuit is associated with displacement currents flowing between the sphere and the interface.

If $b-c$ is made extremely small, the properties of the first medium disappear from eq (5), which reduces to the admittance of a sphere with no dielectric coating. The admittance would then be very large because of the low impedance $\eta_{2}$ of the ionized medium, so that the slot would be virtually shortcircuited. ${ }^{5}$ This makes evident the need for an insulating covering of the sphere. We shall consider, therefore, only the case where the dielectric coating is thick enough to be completely effective in reducing the shunting effect of the ionized sheath across the slot; that is, we assume that $\left|k_{2}\right|(b-a)>>\left(\mu_{2} / \mu_{1}\right) k_{1}^{2} a b$. This is equivalent to

$$
\left|\eta_{2}\right| \ll \frac{b-a}{\omega \epsilon_{1} a b} .
$$

${ }^{5}$ Also, the assumed voltage distribution might not be realized in this case. 
The requirement above on the size of $b-a$ can be interpreted as requiring that this spacing between the plates of the equivalent capacitor be large enough so the capacitive reactance is large compared to the complex impedance of the ionized medium. In other words, we require the spacing to be large enough to prevent the surface currents on the sphere from being short-circuited by displacement currents flowing to the ionized sheath.

Under this stipulation, which is given quantitatively by eq (16), the final expression for $Y$ becomes

$$
Y \approx \frac{1}{\eta_{2}+i \omega \mu_{1}(b-a)} \sum_{n=1}^{\infty} \sum_{m=1}^{n} \pi \frac{m^{2}}{n(n+1)} \bar{P}_{n}^{m}(0)^{2}\left|\frac{V_{m}}{V(0)}\right|^{2} .
$$

This was obtained under the assumptions that

$$
\begin{gathered}
k_{1} b \ll 1 \\
\left|k_{2}\right| b \gg 1 \\
\frac{b-a}{\omega \epsilon_{1} a b} \gg\left|\eta_{2}\right| \\
\frac{b-a}{a} \ll 1 .
\end{gathered}
$$

Since the slot length is small compared to the wavelength, a triangular voltage distribution will be assumed. In this case the coefficients in the Fourier cosine series expansion of $V(\phi)$ are

$$
\begin{aligned}
V_{0} & =\frac{l}{2 \pi a} V(0) \\
V_{m} & =\frac{4 a}{\pi l} \frac{\sin ^{2} \frac{m l}{2 a}}{m^{2}} V(\dot{0}) .
\end{aligned}
$$

Using this distribution, the summation in eq (17) is dependent only on the ratio $l / a$ (through the factor $\left.V_{m}^{2} / V^{2}(0)\right)$, where $l$ is the half-length of the slot and $a$ is the radius of the sphere. The double summation converges approximately as $1 / n^{2}$ and $1 / m^{2}$ (since $V_{m}^{2} / V^{2}(0)$ varies as $1 / m^{4}$ for the assumed triangular voltage distribution), so the series may be evaluated numerically without retaining an extreme number of terms. For a slot of length $l / a=\pi / 2$, the results are roughly

$$
\begin{aligned}
\left.Y\right|_{l / a=\pi / 2} & \approx 0.38 \cdot \frac{1}{\eta_{2}+i \omega \mu_{1}(b-a)} \\
Z & \approx 0.26\left[\eta_{2}+i \omega \mu_{1}(b-a)\right] \\
& \approx 0.26\left\{\frac{\left|n_{2}\right|}{\sqrt{2}}+i\left[\omega \mu_{1}(b-a)+\frac{\left|\eta_{2}\right|}{\sqrt{2}}\right]\right\} .
\end{aligned}
$$

\subsection{Radiation Field}

When the various approximations listed in (18) are used, and in addition, when it is assumed the outer radius, $c$, of the plasma sheath is small compared to a wavelength, the radiation field is found to be due only to the $T E_{11}$ ( $H$-type) mode. Other modes can be neglected because they all contain higher powers of the quantity $k_{3} c$ as factors. The radiation field due to the $T E_{11}$ mode has the form

$$
\begin{aligned}
& E_{\theta}^{(3)} \approx-\frac{3}{4} i k_{3} c \frac{\eta_{2} V_{1}}{\eta_{2}+i \omega \mu_{1}(b-a)} e^{-i k_{2}(c-b)} \frac{e^{-i k_{3} r}}{r} \cos \phi \\
& E_{\phi}^{(3)} \approx \frac{3}{4} i k_{3} c \frac{\eta_{2} V_{1}}{\eta_{2}+i \omega \mu_{1}(b-a)} e^{-i k_{2}(c-b)} \frac{e^{-i k_{3} r}}{r} \cos \theta \sin \phi
\end{aligned}
$$

The electric field has components in both the $\theta$ and $\phi$ directions. These differ only in their dependence on the $\theta$ and $\phi$ coordinates. If a transformation is made to new coordinates $\theta^{\prime}, \phi^{\prime}$, as shown in figure 2, where the new polar angle $\theta^{\prime}$ is measured from the $y$-axis, and the new azimuthal angle $\phi^{\prime}$ is measured from the $z$-axis, then the electric field has only a single component, given by

$$
E_{\phi^{\prime}}^{(3)}=-\frac{3}{4} i k_{3} c \frac{\eta_{2} V_{1}}{\eta_{2}+i \omega \mu_{1}(b-a)} e^{-i k_{2}(c-b)} \frac{e^{-i k_{3} r}}{r} \sin \theta^{\prime} .
$$

The field pattern is now given by the factor sin $\theta^{\prime}$. This is exactly the pattern of a loop lying in the $x z$-plane with center at the origin. This is the meridian plane of the currents which flow from one edge of the slot around the sphere to the opposite edge of the slot.

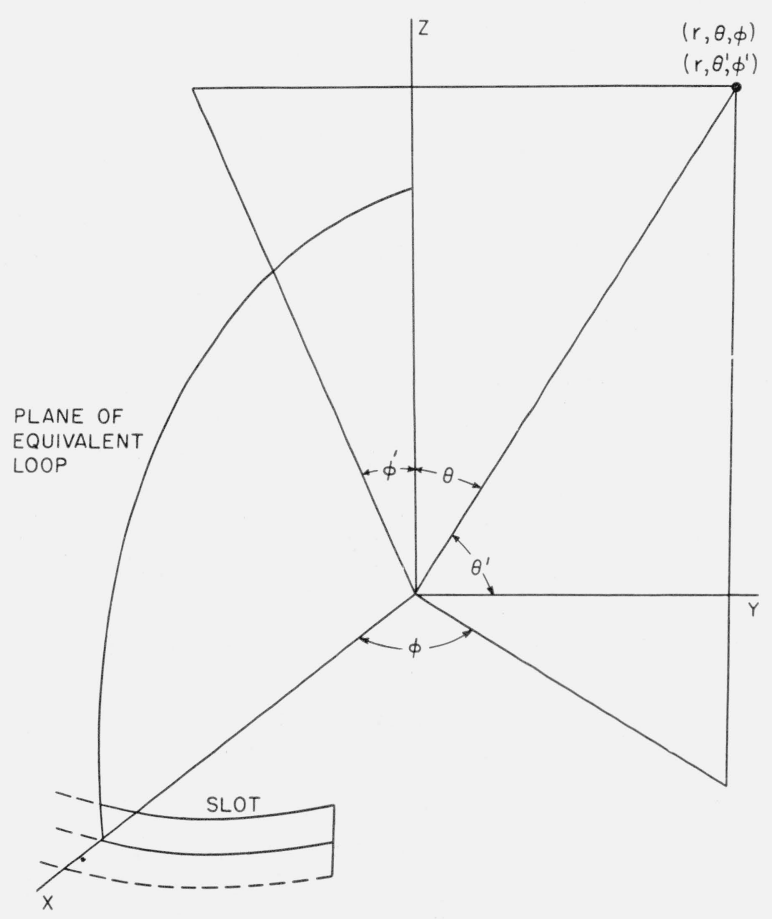

Figure 2. Slotted-sphere antenna and equivalent loop. 


\subsection{Radiation Intensity and Input Power}

From eq (22), it is easy to calculate the power radiated per unit solid angle, or the radiation intensity.

The radiation intensity is

$\frac{r^{2}\left|E_{E \phi^{\prime}}^{(3)}\right|^{2}}{2 \eta_{3}}=\left(\frac{3}{4} k_{3} c\right)^{2}\left|\frac{\eta_{2}}{\eta_{2}+i \omega \mu_{1}(b-a)}\right| \frac{{ }^{2}}{2 \eta_{3}} e^{2} e^{-\sqrt{2}\left|k_{2}\right|(c-b)} \sin ^{2} \theta^{\prime}$.

The total input power, $P$, which includes both the power radiated and the power absorbed by the sheath, is given by the real part of $Y V(0)^{2} / 2$. From eq (17) this is found to be

$P=\frac{1}{2 \sqrt{2}} \frac{\left|\eta_{2}\right|}{\left|\eta_{2}+i \omega \mu_{1}(b-a)\right|^{2}} \sum_{n=1}^{\infty} \sum_{m=1}^{n} \pi \frac{m^{2}}{n(n+1)} \bar{P}_{n}^{m}(0)^{2} V_{m}^{2}$

\subsection{External Efficiency}

A measure of the efficiency of the antenna can be obtained by calculating the external efficiency,or ratio of the radiated power to the input power supplied to the slot. This ratio then can be examined for an optimum choice of the design parameters. This, however, is only a partial optimization procedure, since it does not consider all the factors which determine the overall efficiency of the antenna. In particular, it does not include the losses in the matching networks necessary to feed the slot from the source of power. These losses will depend on the $Q$ of the matching network and the input impedance. This latter problem, however, can be evaluated since the input impedance of the slot already has been determined.

Denoting the external efficiency by $\xi$,

$$
\xi=\int_{-\pi}^{\pi} \int_{0}^{\pi} \frac{\left.||_{E \phi^{\prime}}^{(3)}\right|^{2}}{2 \eta_{3} P} r^{2} \sin \theta^{\prime} d \theta^{\prime} d \phi^{\prime}
$$

Substituting (23) and (24) into (25), and integrating

$$
\begin{gathered}
\xi=\left[\begin{array}{c}
\pi \frac{m^{2}}{n(n+1)} \bar{P}_{n}^{m}(0) \frac{V_{m}^{2}}{V(0)^{2}} \mid \begin{array}{c}
m=1 \\
n=1
\end{array} \\
\sum_{n=1}^{\infty} \sum_{m=1}^{n} \pi \frac{m^{2}}{n(n+1)} \bar{P}_{n}^{m}(0) \frac{V_{m}^{2}}{V(0)^{2}}
\end{array}\right] \\
\times\left[\left(k_{3} c\right)^{2} \frac{4 \sqrt{2}\left|\eta_{2}\right|}{\eta_{3}} e^{-\sqrt{2}\left|k_{2}\right|(c-b)}\right] .
\end{gathered}
$$

Each of the brackets in (26) has physical significance. The first bracket is the ratio of the power delivered to the magnetic type mode $n=m=1$ $\left(T E_{11}\right)$, (which is the only mode contributing signifi- cantly to the radiation field) to that delivered to all the modes. The second bracket is the overall power transmission coefficient for this mode (i.e., the ratio of power of this mode that is radiated to that entering the sheath). It consists of an exponential factor representing attenuation through the sheath, and a second factor due to reflection at the outer surface of the sheath. The latter is the product of $\left(k_{3} c\right)^{2}$ and the factor $4 \sqrt{2}\left|\eta_{2}\right| / \eta_{3}$ which in turn is the power transmission coefficient for a plane wave progressing across a plane surface from a medium of intrinsic impedance $\eta_{2}$ to one whose intrinsic impedance is $\eta_{3}$ (with $\left|\eta_{2}\right|<<\eta_{3}$ ).

\section{Optimization Procedures}

The expression above is proportional to the efficiency (ratio of radiated power to total input power) of the slot antenna if it is assumed that feeding losses are not important compared to losses in the sheath. Therefore, it is desirable to consider the means for maximizing the expression given in eq (26). In the first place, if the size of the vehicle is given, the quantities $b$ and $c$ are fairly well fixed. Since the outer medium is free space, $\eta_{3}$ will be equal to 377 ohms. The quantity $k_{3}^{2} \equiv \omega^{2} \mu_{3} \epsilon_{3}$ varies as the square of the frequency while $\left|\eta_{2}\right|=\sqrt{\omega \mu_{2} / \sigma}$ and $\left|k_{2}\right|=\sqrt{\omega \mu_{2} \sigma}$ vary as the square-root of the frequency. Consequently $\xi$ varies as $\omega^{5 / 2} \exp$ $\left[-\sqrt{2 \mu_{2} \sigma}(c-b) \omega^{1 / 2}\right]$. The latter expression is maximized by setting the exponent equal to -5 :

$$
\sqrt{2 \mu_{2} \sigma}(c-b) \omega_{0 p t}^{1 / 2}=5 .
$$

The optimum frequency then is

$$
f_{0 p t}=\frac{25}{4 \pi} \frac{1}{\mu_{2} \sigma(c-b)^{2}} .
$$

This is the frequency that makes the sheath thickness, $c-b$, equal to two-and-one-half skin depths in the ionized medium.

The only other quantity in (26) besides the frequency that can be varied to make the expression a maximum is the ratio

$$
\frac{\pi \frac{m^{2}}{n(n+1)} \bar{P}_{n}^{m}(0)^{2} \frac{V_{m}^{2}}{V(0)} \mid \begin{array}{c}
m=1 \\
n=1
\end{array}}{\sum_{n=1}^{\infty} \sum_{m=1}^{n} \pi \frac{m^{2}}{n(n+1)} \bar{P}_{n}^{m}(0)^{2} \frac{V_{m}^{2}}{V(0)^{2}}} .
$$

The magnitude of this can be varied by changing the length of the slot. In general, the efficiency is increased by increasing the slot length.

The thickness, $b-a$, of the dielectric layer does not appear in the expression (26) for the efficiency provided condition (16) is met. It does, however, appear in (20) where it is present as a term representing an additional series inductance. 


\section{Appendix}

A situation that is possibly of greater practical interest than the entirely insulated spherical antenna treated above is the case of the partially insulated slotted sphere, one octant of which is illustrated in figure 3. The angular width of the dielectric cover is the same as the angular width of the slot and is equal to $2 \phi_{0}$. The angular height of the slot is $2 \psi_{0}$. The outer surface of the dielectric is flush with the surface of the conducting sphere. The input impedance of this configuration was estimated [9] (for low frequencies) by calculating the input admittance of a rectangular parallelepiped obtained by straightening the curved dielectric slab. The result was

$$
Y= \begin{cases}\frac{1}{3} \frac{1}{\eta_{2}+i \omega \mu_{1}(b-a)} \frac{1+\left(\frac{\psi_{0}}{\phi_{0}}\right)^{2}-0.23\left(\frac{\psi_{0}}{\phi_{0}}\right)^{3}}{\left(\frac{\psi_{0}}{\phi_{0}}\right)} ; 0<\frac{\psi_{0}}{\phi_{0}}<1.5 \\ \frac{1.63}{3} \frac{; 1.5<\frac{\psi_{0}}{\eta_{0}} .}{\eta_{2}+i \omega \mu_{1}(b-a)} & \text {. }\end{cases}
$$

The external efficiency resulting in this case was also derived. The attenuation and reflection losses are given in $\mathrm{db}$ by

$$
10 \log \left[\left(k_{3} c\right)^{2} \frac{4 \sqrt{2}\left|\eta_{2}\right|}{\eta_{3}} e^{-\sqrt{2}\left|k_{2}\right|(c-b)}\right]
$$

where the bracket is, of course, the same as the second one in eq (26). The loss to higher order modes is given by a different expression, however, and is plotted in figure 4.

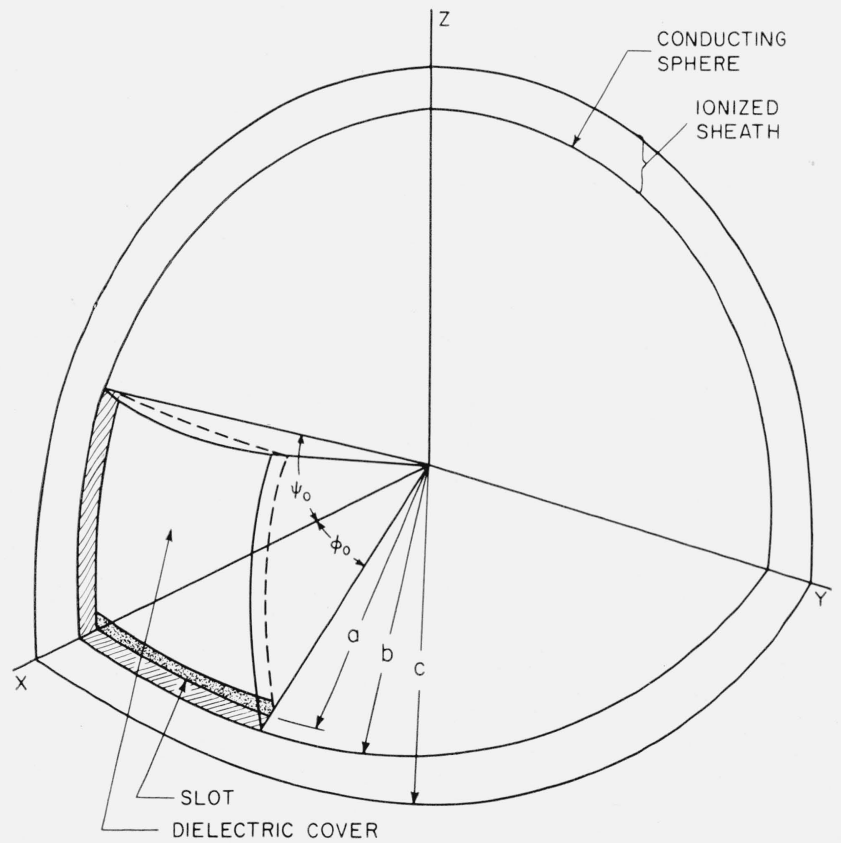

Figure 3. Partially insulated slotted-sphere antenna.

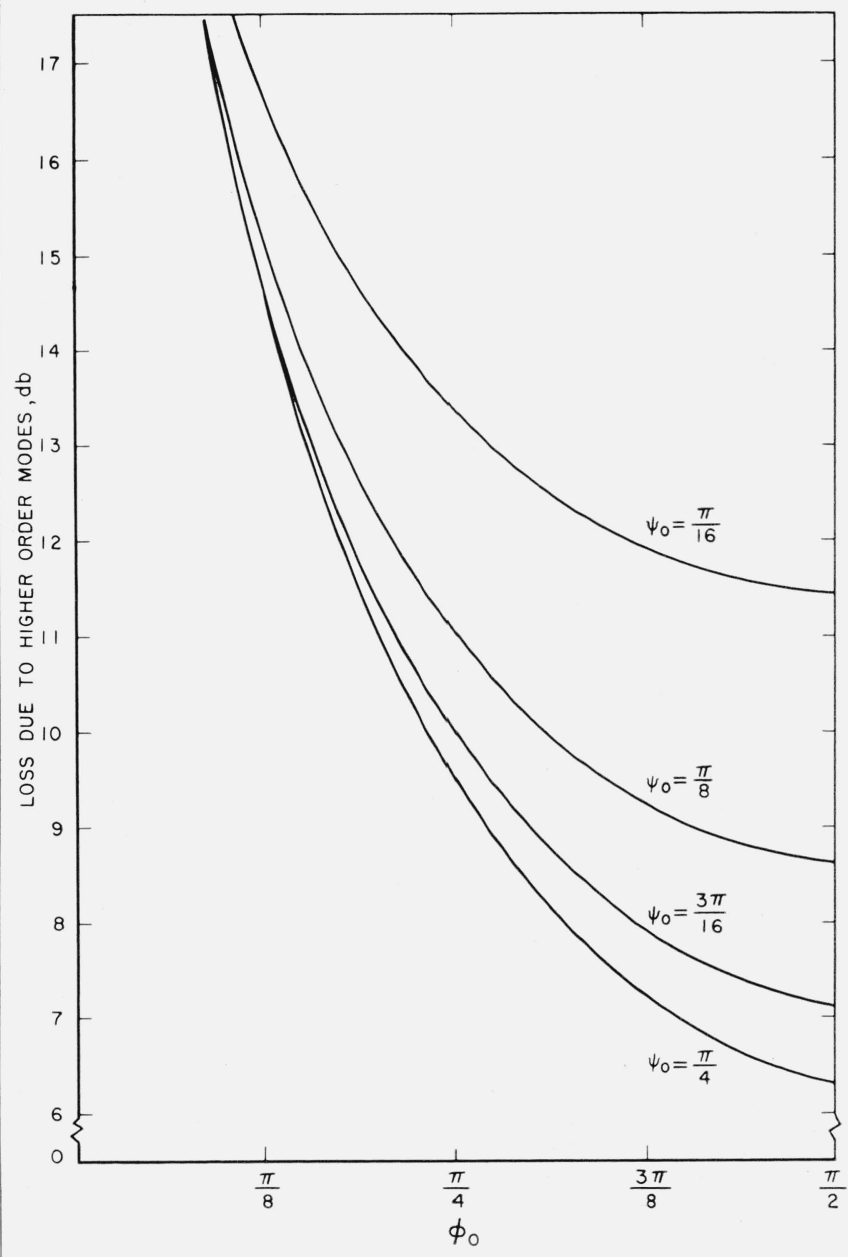

Figure 4. Higher order mode loss for the partially insulated slotted-sphere antenna.

\section{References}

[1] J. R. Wait, Electromagnetic radiation from cylindrical structures (Pergamon Press, Inc., New York, N.Y., 1959).

[2] Y. Mushiake and R. E. Webster, Radiation characteristics with power gain for slots on a sphere, IRE Trans. on Antennas and Propagation AP-5, 47 (1957).

[3] Martin Katzin, Radiation of an electric dipole into an ionized sheath, Electromagnetic Research Corp., Rept. 60175-7 (15 Dec. 1957).

[4] J. A. Ratcliff, The magneto-ionic theory and its application to the ionosphere, eq 4.2.6, and para. 1.5 (Cambridge University Press, Cambridge, England, 1959).

[5] J. A. Ratcliff, op. cit., eq 4.4.1.

[6] L. I. Bailin and S. Silver, Exterior electromagnetic boundary value problems for spheres and cones, IRE Trans. on Antennas and Propagation AP-4, 5 (1956).

[7] Jahnke and Emde, Tables of functions, ch. VII, para. 7, p. 116 (Dover Publications, New York, N.Y., 1951).

[8] S. A. Shelkunoff, Electromagnetic waves, para. 3.5 (D. Van Nostrand Co., Inc., New York, N.Y., 1943).

[9] J. W. Marini, B. Y.-C. Koo, and M. Katzin, Radiation and impedance of antennas surrounded by an ionized sheath, Electromagnetic Research Corporation, Report ERC 60301-6 (25 Aug. 1958). 